\title{
Design of a smart sensor mesh for the measurement of pH in ostomy applications
}

Anna McLister ${ }^{1}$ (D), Charnete Casimero ${ }^{1}$ (D), Aaron McConville ${ }^{1}$ (D), Charlotte M. Taylor $^{2}$ (D), Clare L. Lawrence ${ }^{3}$ (D), Robert B. Smith ${ }^{2}$ (D), Ashish Mathur ${ }^{4}$ (D), and James Davis ${ }^{1, \star}$ (D)

${ }^{1}$ School of Engineering, Ulster University, Jordanstown, Northern Ireland BT37 OQB, UK

${ }^{2}$ Chemistry, School of Physical Sciences and Computing, University of Central Lancashire, Preston PR1 2HE, UK

${ }^{3}$ School of Pharmacy and Biomedical Sciences, Faculty of Clinical and Biomedical Sciences, University of Central Lancashire, Preston PR1 2HE, UK

${ }^{4}$ Amity Institute of Nanotechnology, Amity University, Noida, Uttar Pradesh 201313, India

Received: 18 January 2019

Accepted: 4 April 2019

Published online:

15 April 2019

(C) The Author(s) 2019

\begin{abstract}
A carbon-loaded polyethylene film was modified through a combination of laser ablation and electrochemical anodisation to yield a mechanically flexible yet electroanalytically sensitive mesh. A custom flavin derivative bearing a pendant phenol substituent was electropolymerised onto the substrate, and its electrochemical properties were investigated. The reversible flavin electrochemistry was retained $(\mathrm{Ep}=-0.374 \mathrm{~V}$ vs. $\mathrm{Ag} / \mathrm{AgCl}, \mathrm{pH} 7)$, and the peak position was found to shift by $60 \mathrm{mV} / \mathrm{pH}$ over a range covering $\mathrm{pH} 2.55$ to $\mathrm{pH} 8.12$. The stability of the resulting composite has been evaluated, and the analytical applicability towards the voltammetric measurement of $\mathrm{pH}$ in human urine was critically assessed.
\end{abstract}

\section{Introduction}

Surgical intervention in which bowel or bladder function is diverted through an abdominal stoma is life-changing event, and it has been estimated that the patient population in the USA alone is in the million range. Despite many advances in the production of ostomy management systems, a significant number of patients continue to experience complications post-surgery [1-5]. Inflammation and erosion of the skin surrounding the stoma site is remarkably common and can arise through a number of factors but, in the overwhelming majority of cases, it can be attributed to prolonged exposure to urine or stool [1-5]. Effluent from the stoma is collected through the use of a pouch system (Fig. 1a) which is attached to the abdomen through an adhesive skin wafer (faceplate) placed over the stoma [6, 7]. In principle, the wafer should act as a physical barrier preventing the stoma output from contacting the underlying skin, but, sadly, it can all too often fail leading to maceration of the skin (Fig. 1b) and the onset of irritation and ulceration [6-8]. The situation can be greatly exacerbated through exposure to high $\mathrm{pH}$ effluent

Address correspondence to E-mail: james.davis@ulster.ac.uk 


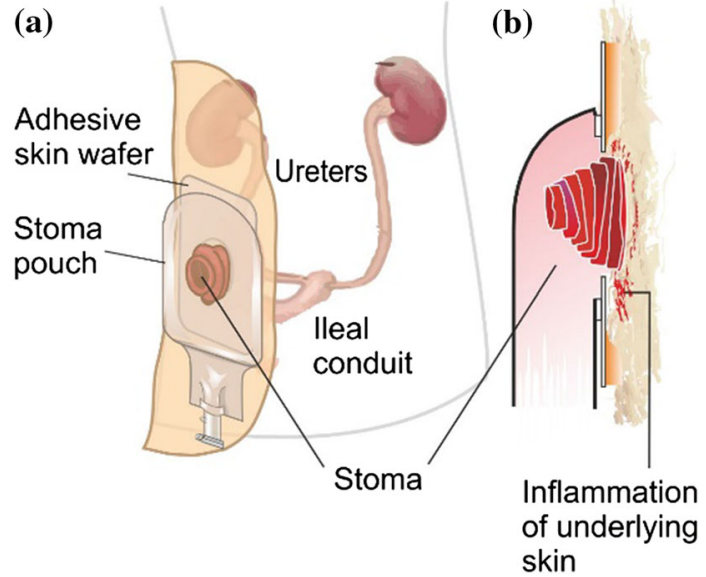

Figure 1 a Typical application of pouch appliance. $\mathbf{b}$ Cross section of pouch placement highlighting zone where inflammation occurs.

which can compromise the skin's barrier function [9-13]. Moreover, it has long been recognised that urine $\mathrm{pH}$ can be a useful diagnostic handle for monitoring kidney function [14-17], a critical issue particularly for those dealing with the physical trauma associated with a urostomy. The aim of the present communication has been to examine the electrochemical performance of a mechanically flexible polymeric mesh modified with a novel redox polymer and assess its ability to function as a $\mathrm{pH}$ sensor and which could ultimately be integrated within a new generation of smart skin wafers.

There is an extensive literature on the development of $\mathrm{pH}$ sensing systems for monitoring urine $\mathrm{pH}$ and covers a wide variety of methodologies such as luminescence imaging [18], fibre-optic probes [19], near-infrared reflectance spectroscopy [20], absorption and fluorescence spectroscopy [21] and magnetic resonance spectroscopy [22]. Electrochemical techniques, however, predominate [23-32] and can be more amenable to periodic or continuous monitoring in situ [29-32]. This is especially relevant when considering the compositional complexity and heterogenous nature (volume, surface area or shape) of the sample matrix. It is only in recent years that there has been an interest in flexible/wearable systems [29-32] and, in the case of ileostomy and urostomies, there is a need for a mouldable membrane type sensor that is unobtrusive to the patient. The need for device disposability creates further difficulties in the design process where the manufacture of low cost yet robust and reproducible sensing structures is required.

The foundations of the proposed sensor are based on a polyethylene film doped with carbon particles (C-PE) to confer electrically conductivity. The carbon-polymer composite is a commercial variant originally designed to serve as an electromagnetic screening film [33], but it was envisaged that through removing the outermost polymer layers and exposing a greater proportion of interfacial carbon, the electroanalytical sensing performance could be improved [30]. A mesh design was proposed whereby laser processing could be employed to etch tracks within the film at a level insufficient to penetrate through the film. It could be anticipated that were the tracks rastered across the $X$ and $Y$ planes, a hole would be created where the tracks intersected and thereby effectively create a porous mesh-like structure. While such modifications could be expected to yield a conductive and mechanically mouldable film, it was noted that the underlying carbon would fail to possess any inherent analytical selectivity. Given that the principal aim was to develop a mesh capable of monitoring the $\mathrm{pH}$ of bodily fluids, it was therefore necessary to consider a further modification to the base system.

The oxides of metals such as tungsten [18], manganese [19], ruthenium [20], iridium [21] and zinc [22] have long been exploited as $\mathrm{pH}$ sensitive components in the design of sensors, and there continues to be much interest their nanostructured analogues [22]. More recent developments have seen graphene and graphene oxide come to the fore as potential $\mathrm{pH}$ sensors [23, 24]. In recent years, there has also been a steady interest in the use of voltammetric approaches where the peak positions arising from either the oxidation or reduction in indicator molecules (bearing quinoid [25, 26] or nitro [27] functionality) are used as an indirect measure of the local $\mathrm{pH}$. In this instance, we sought to investigate the modification of the laser exposed carbon particles with a novel flavin type molecular species (Fig. 2, Structure I). Flavins are known to exhibit near-reversible electrochemical behaviour [34] which, by virtue of the intrinsic acidbase functionality of the core structure, will be $\mathrm{pH}$ dependent. The key challenge in the proposed design, however, relates to anchoring the redox system to the exposed carbon particles within the composite mesh. The custom flavin detailed in Fig. 2 possesses an electropolymerisable phenol group 


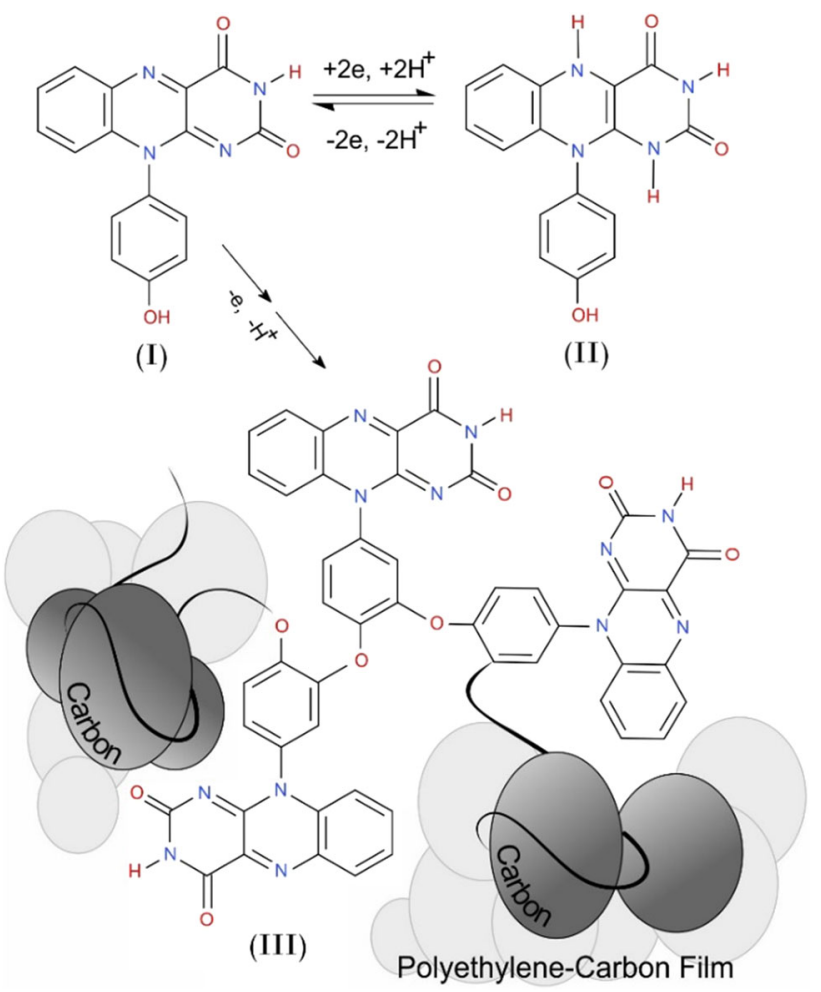

Figure 2 Redox transition of the flavin unit $(\mathrm{I} \rightarrow$ II) and the electro-oxidation of the phenolic substituent leading to the production of a polyphenylene oxide polymer $(\mathrm{I} \rightarrow \mathrm{III})$ on exposed carbon surfaces at the film interface.

which, it was envisaged, after electro-oxidation would lead to film formation and thereby trap the $\mathrm{pH}$ sensitive redox group at the electrode. The electropolymerisation process has been successfully demonstrated with carbon fibre and screen-printed systems and found to preserve the activity of the flavin centre [35, 36]. The key molecular transitions are highlighted in Fig. 2.

The preparation of the C-PE composite mesh and its subsequent modification with the poly flavin film was therefore investigated, and its potential for use a $\mathrm{pH}$ sensing film was critically assessed through examining the response in urine.

\section{Experimental details}

All chemicals were obtained from Sigma-Aldrich, were the highest grade available and were used without further purification. Electrochemical analysis was carried out using a VSP-300 Multichannel Potentiostat/Galvanostat/EIS (Bio-Logic Science Instruments, EC-Lab Ltd) with a standard three- electrode configuration with either a glassy carbon or carbon-polyethylene composite as the working electrode. Platinum wire served as the counter electrode and a conventional silver/silver chloride ( $3 \mathrm{M} \mathrm{NaCl}$, BAS Technicol, UK) reference electrode. All measurements were conducted at $22 \pm 2{ }^{\circ} \mathrm{C}$. BrittonRobinson buffers (acetic, boric and phosphoric acids, each at a concentration of $0.04 \mathrm{M}$ and adjusted to the appropriate $\mathrm{pH}$ through the addition of sodium hydroxide) were used throughout unless otherwise specified. Butyl-grafted polyethylene films filled with carbon black were supplied by Goodfellow Research Materials ( $80 \mu \mathrm{m}$ thick, resistivity of $100 \Omega \mathrm{cm}$ ).

\section{Preparation/characterisation of modified films}

Laser patterning of the polymer was achieved using a 30-W $\mathrm{CO}_{2}$ air-cooled computer-controlled laser cutter (FB400 series CadCam Technology Ltd, Nottingham, UK). Directional control over the laser, raster/vector speed and output power was by means of the proprietary software (ApS-Ethos) with a spatial resolution of 25 micron. The laser was rastered across the $x$ and $y$ directions with a spacing of $2 \mathrm{~mm}$ between each pass. The surface profile and etch depths were characterised using a DEKTAK XT Stylus Profiler (Bruker) system with a single pass accounting for a depth of 60 micron. The surface profile is highlighted in Fig. 3 along with an inset photograph detailing the resulting lasered film. Visual inspection of the film easily highlights the porous mesh-like structure of the film and stands in marked contrast to the initial impermeable, featureless film. The pores are due to the intersection of perpendicular laser tracks and can be attributed to the fact that, while one pass alone can etch to a depth of 60 micron, a portion of track receiving a second pass (at the intersection) has the potential to etch a further 60 micron and will therefore effectively burn through the 80 micron film.

The film was also characterised using scanning electron microscopy, and representative images of the resulting mesh are shown in Fig. 4a-c. Examination of the laser tracks reveals a highly porous structure and can be attributed to the ablation of the polyethylene leaving behind residual carbon (Fig. 4b), and this is further exemplified in Fig. 4c where the structure of the pore interface (having received 2 laser passes) is shown. The untreated film (Fig. 4b), in contrast, has a relatively featureless 


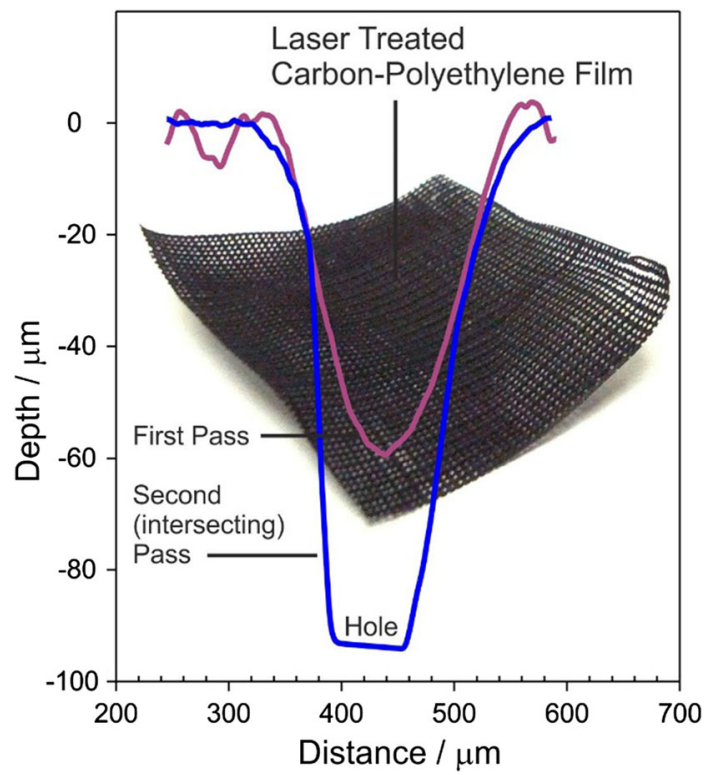

Figure 3 DEKTAK surface profile of the lasered film highlighting the typical depth profile of areas receiving a single and double pass. Insert: photograph of the C-PE film after laser treatment.

morphology in which isolated aggregations of carbon particles can be seen to penetrate the film surface (as previously postulated in Fig. 1). It is clear that the laser treatment greatly increases the exposure of the carbon particles and thus should, along the tracks, increase the interfacial electrochemical properties.

The C-PE film was then sectioned and mounted in a laminate casing with a $4-\mathrm{mm}$ square window and thermally sealed $[28,30]$. The latter was done to enable comparative investigation of the film properties. It has become relatively common to electrochemically anodise carbon composite electrodes in order to elicit improved electrochemical behaviour $[28,30]$. The electro-oxidation $(+2 \mathrm{~V}, 0.1 \mathrm{M} \mathrm{NaOH})$ typically increases exfoliation of the carbon particles-generating more edge plane sites and increases the populations of various oxygen functional groups $[28,30]$. This process was also instituted here where it has been found that the unmodified screen-printed electrodes typically exhibit poor electrochemical behaviour with large overpotentials necessary to obtain any significant analytical responses [30].

\section{Flavin electropolymerisation}

Riboflavin was obtained from commercial sources, whereas the phenol derivative was custom
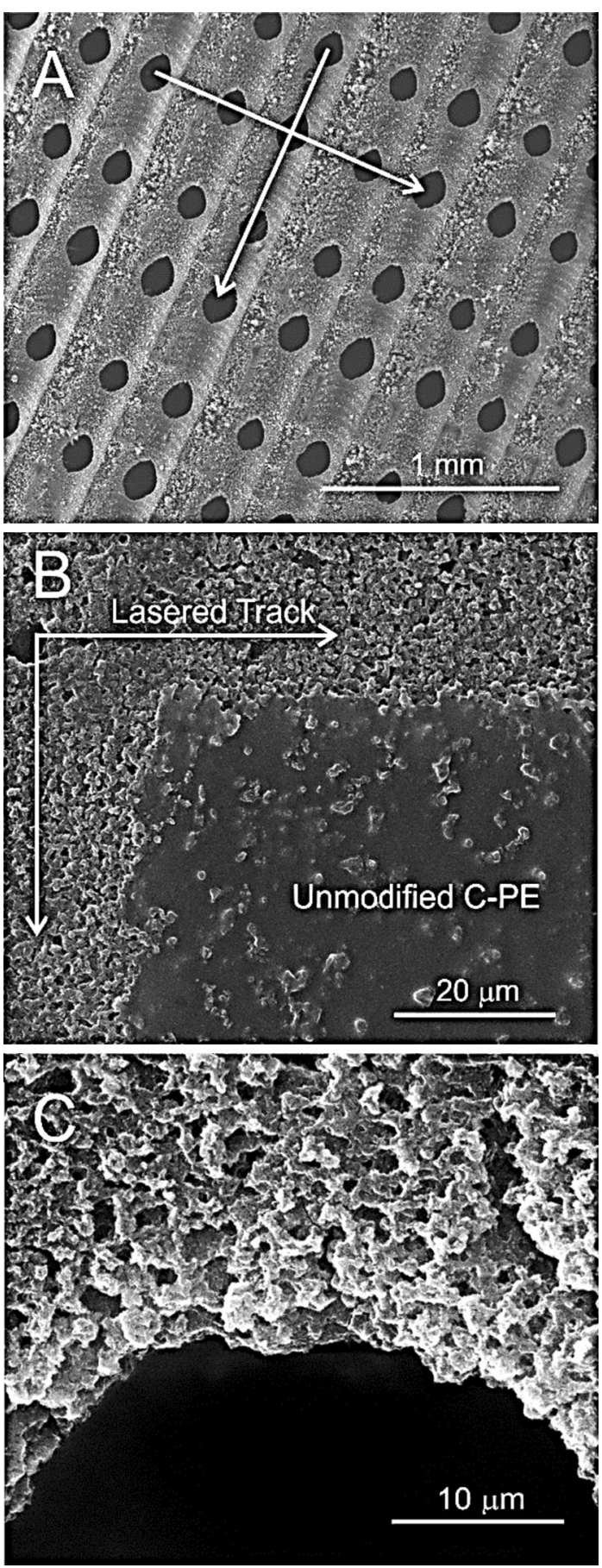

Figure 4 Scanning electron micrographs of the carbonpolyethylene mesh after laser processing. a Low magnification highlighting the direction of the laser raster and creation of holes within the film. b Comparison of the laser-etched track and unmodified film. c Removal of the polyethylene at the pore edge as a consequence of laser double pass.

synthesised. The synthesis of the 10-(4-hydroxyphenyl)benzo[g]pteridine-2, $4(3 \mathrm{H}, 10 \mathrm{H})$-dione was 
accomplished using methods previously published [35]. Electropolymerisation was conducted through placing the carbon-polyethylene film into an aqueous solution containing the phenol derivative $(150 \mu \mathrm{M}$, $\mathrm{pH}$ 7). Repetitive scan cyclic voltammetry $(+0.2 \mathrm{~V} \rightarrow-0.8 \mathrm{~V} \rightarrow+1 \mathrm{~V}, 50 \mathrm{mV} / \mathrm{s})$ was used to initiate the electropolymerisation process. Solutions were generally degassed with nitrogen prior to commencing the experiments and run under nitrogen blanket.

\section{Analytical characterisation/ethics approval and compliance}

The analytical capability of the mesh sensors was assessed using human urine as the test matrix principally to determine the robustness of the material and to assess the possibility of surface passivation from either matrix components or electrode process by-products. Urine was obtained from three healthy volunteers $(2 \mathrm{M}, 1 \mathrm{~F})$ who had not taken any form of medication in the $24 \mathrm{~h}$ preceding collection of the samples. The samples were collected, anonymised, stored at $4{ }^{\circ} \mathrm{C}$ until required and then flushed to waste once the study had concluded. Approval for the use of human urine samples was granted through the Ulster University Ethics Committee (UREC Ref: $\mathrm{REC} / 16 / 0073)$ prior to commencing the investigation.

\section{Results and discussion}

The electropolymerisation of the phenolic flavin derivative onto carbon fibre substrates for use in bacterial reactors has been successfully demonstrated [35], but it was necessary to determine if similar electrochemistry would be exhibited at the lasered composite mesh. It was anticipated that the electrochemical properties of the carbon-polyethylene mesh would be inferior to those exhibited by glassy carbon and carbon fibre given the composite nature of the film structure. It has been shown in Fig. $4 \mathrm{~b}$ that the bare, unmodified film, has a relatively low population of exposed carbon sites through which to induce electroanalytical activity; however, the laser modification should substantially improve performance. This would still leave the carbon as largely graphitic/ basal plane structures, and therefore, the pre-anodisation through electrochemical oxidation in $\mathrm{NaOH}$ should lead to the exfoliation of the former providing greater edge plane sites that should enhance electron transfer. The procedure used to form the flavin on the glassy carbon electrode was again employed for the carbon-polyethylene mesh electrode with the cyclic voltammograms detailed in Fig. 5. While the voltammetric profile is similar in form to those previous reported at glassy carbon electrodes [35], the peak processes lack the sharpness with a greater peak separation attributed to the composite nature of the mesh. Nevertheless, the three-electrode processes indicated in Fig. 2 are clearly recognisable. The reduction and oxidation peaks associated with the flavin component (I > II and II > I) can be seen at $-0.65 \mathrm{~V}$ and $-0.1 \mathrm{~V}$, respectively. This initial behaviour is consistent with the response observed by others when examining riboflavin $[37,38]$ at carbon-based electrodes. However, the voltammetric profile changes significantly upon scanning towards more positive potentials. The oxidation of the phenolic component $(\mathrm{I}>\mathrm{III})$ can be seen at $+0.81 \mathrm{~V}$ and decreases with consecutive scans and is consistent with the behaviour expected of mono-phenolic species.

It was envisaged that the oxidation leads to the formation of oligomeric and polymeric deposits as indicated in Fig. 2. When the scan direction is reversed and the potential is swept back towards the

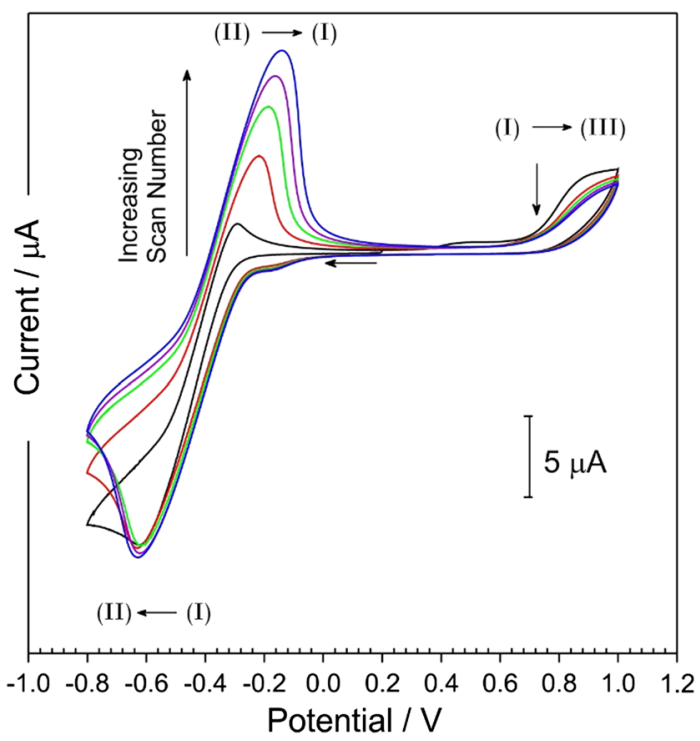

Figure 5 Cyclic voltammograms detailing the response of the carbon-polyethylene mesh electrode towards $2 \mathrm{mM}$ ferrocyanide $(0.1 \mathrm{M} \mathrm{KCl})$ before and after various surface modifications. Scan rate: $50 \mathrm{mV} / \mathrm{s}$. 
flavin reduction region however, the magnitude of both flavin peak processes is significantly increased. In separate studies involving the phenolic compound, repetitively scanning the flavin region without inducing the oxidation of the phenol did not lead to any change in the peak magnitudes. Thus, the increase in the peak height observed on the second and subsequent scans shown in Fig. 5 can therefore be attributed to the formation of the polymeric deposit on the electrode surface and the accumulation of the material at the interface as anticipated in Fig. 2.

The electrode was removed, rinsed and placed in fresh buffer. Rather than using cyclic voltammetry to assess the response to $\mathrm{pH}$ and perform the measurements under nitrogen, square wave voltammetry was employed to enhance the peak profile without any prior degassing of the solution. Representative voltammograms obtained at the flavin modified mesh are detailed in Fig. 6. The position of the oxidation peak was found to vary with $\mathrm{pH}$ in an analogous manner to that observed with the glassy carbon electrode with a $60 \mathrm{mV} / \mathrm{pH}$ shift (E/ $\left.V=0.060 \mathrm{pH}+0.0972 ; N=7 ; R^{2}=0.998\right)$. A critical factor here is that although the peak potentials are within a negative potential region, the response was found to be unaffected by the presence of oxygen.

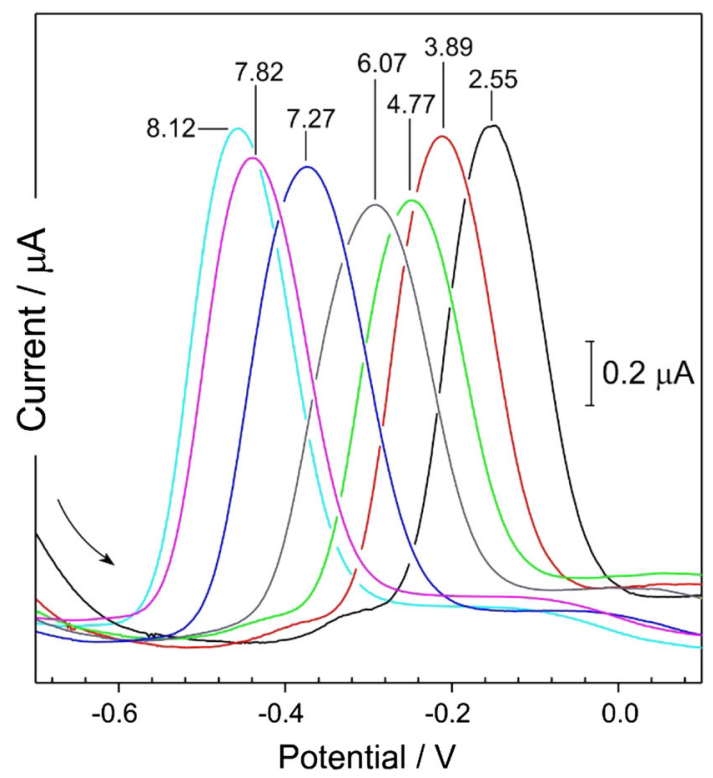

Figure 6 Square wave voltammograms detailing the response of a poly flavin modified carbon-polyethylene mesh electrode in Britton-Robinson buffers of varying $\mathrm{pH}$.
The ability of the film to repeatedly monitor $\mathrm{pH}$ was determined through running the C-PE/flavin film through a sequence of Britton-Robinson buffer solutions ( $\mathrm{pH} 2.55$ to $\mathrm{pH} 8.12$ ) that covered a physiologically relevant range. A sequence of three measurement series was completed to confirm that the $\mathrm{pH}$ response at the electrode was reversible and to assess the degree of drift. Each $\mathrm{pH}$ buffer was scanned three times giving rise to a total of 63 scans over the entire three series. The variation of peak position with $\mathrm{pH}$ for each run is highlighted in Fig. 7a.

It should be noted that each point is the average of three measurements and although error bars are included within the figure-the variation (typically less than $2 \mathrm{mV}$ ) is so small that they are barely discernible from the actual marker. The influence of this repetitive scanning on the peak height can also give some indication of the stability of the flavin film. The normalised peak height (based on the magnitude of the first scan) as a function of scan number is highlighted in Fig. 9b. It can be seen that over 63 repetitive scans (and over 3 entire $\mathrm{pH}$ scan series), the oxidation peak associated with the flavin decreases by less than $20 \%$. This is a crucial factor given that the original intention was to engineer a film that could periodically monitor the $\mathrm{pH}$ of a biofluid within a device such as a dressing, catheter or stoma where the device would, ideally, need to be in place for several days [1-5].

A key attribute of the carbon-loaded polyethylene is that it should be mechanically flexible in order to conform to the morphology of the skin. It was therefore necessary to determine the effect of bending on the electroanalytical performance of the film. The polymer was electropolymerised onto the mesh as described previously and square wave voltammetry used to probe the response (peak position and peak height) of the flavin redox centre. The films were subjected to mechanical flexing and the voltammograms recorded. In general, a total of 50 flexing cycles (at $90^{\circ}$ ) were performed and electrochemical response recorded after every fifth cycle. Square wave voltammograms detailing the influence of repetitive bending on the flavin peak process is highlighted in Fig. 8a. A second type of experiment was also conducted where the film was initially scanned and then bent at $90^{\circ}$ and held fixed (folded) in that position. The corresponding voltammograms are compared in Fig. $8 \mathrm{~b}$. 

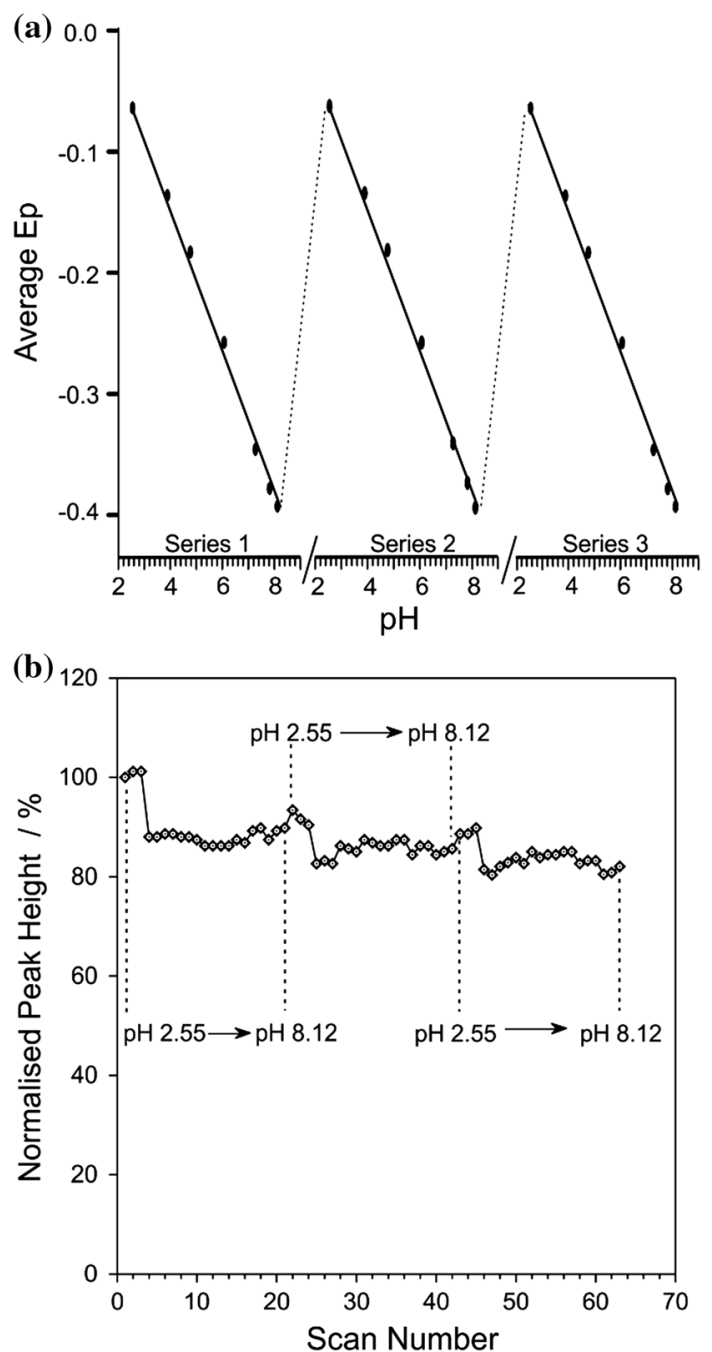

Figure 7 a Variation of peak potential with $\mathrm{pH}$ over three consecutive $\mathrm{pH}$ series recorded at the flavin modified carbonpolyethylene mesh. $\mathbf{b}$ Influence of repetitive cycling and $\mathrm{pH}$ on the peak magnitude of the flavin modified carbon-polyethylene mesh.

Repetitive binding did not lead to catastrophic failure, and the flavin response was observed after the film has returned to its plane configuration. The fact that the flavin was also observed when held at $90^{\circ}$ also attests to the inherent conductivity of the material upon deformation. It can be seen from Fig. 8a however that the magnitude of the flavin peak process decreases with each cycle. A similar observation is seen in Fig. $7 \mathrm{~b}$ where repetitive scanning (in the absence of mechanical flexing) also leads to a decrease in peak current-albeit at a reduced rate. A more quantitative evaluation of peak position and peak height with bend cycle is detailed in Fig. 9a, b, respectively. (a)

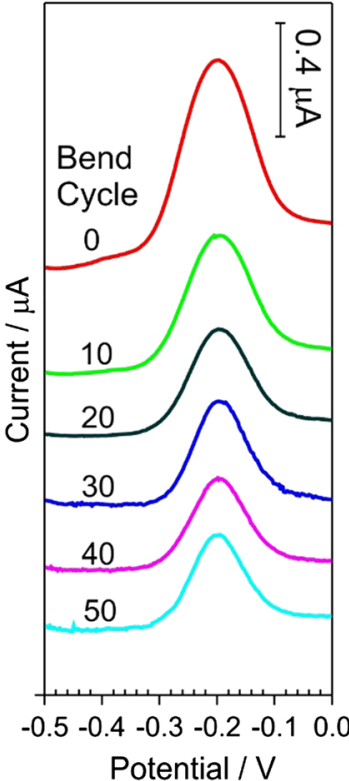

(b)

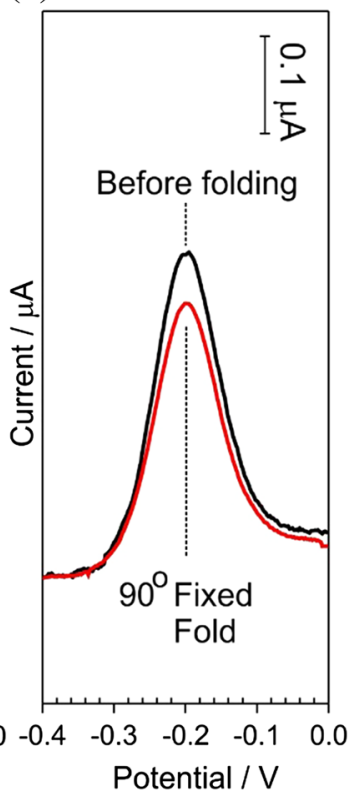

Figure 8 Square wave voltammograms detailing the response of the flavin modified carbon-polyethylene film in pH 5 BrittonRobinson buffer after a repetitive bend $\left(90^{\circ}\right)$ cycles and $\mathbf{b}$ after being fixed at $90^{\circ}$.

The critical point to note from Fig. 8 is that while the peak magnitude decreases (possibly as material is lost from the surface), the peak position remains constant (\%RSD $=0.6 \% ; N=50)$ throughout both the mechanical flexing and when the film is fixed in a $90^{\circ}$ bend. The analytical signal is derived not from the peak magnitude but from the peak position, and

(a)

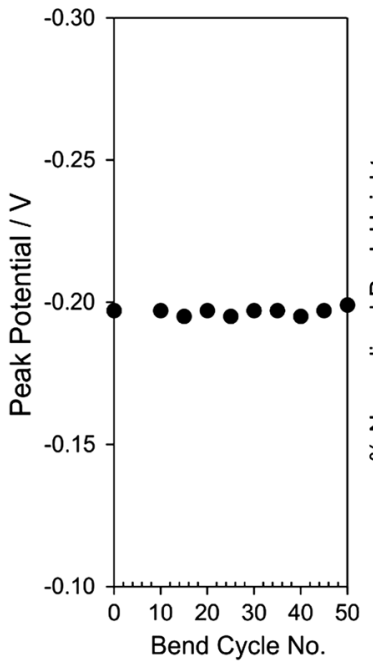

(b)

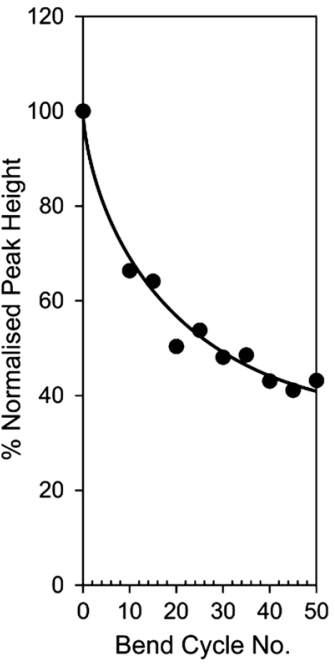

Figure 9 Variation of the poly flavin peak potential (a) and peak height (b) with repetitive bending $\left(90^{\circ}\right)$ motions. 
thus, the latter's insensitivity to mechanical flexing is a significant advantage.

The analytical accuracy of the film was assessed through comparing the response in human urine with a conventional glass $\mathrm{pH}$ probe (Hanna). Urine was obtained from three healthy volunteers immediately prior to conducting the analysis. The C-PE/ flavin mesh was immersed in the urine sample, and three square wave voltammograms were recorded (analogous to the process used previously and highlighted in Fig. 6). A representative voltammogram of the C-PE/flavin mesh in urine is detailed in Fig. 10 and highlights the unambiguous identification of the flavin oxidation peak. A second, broader peak process can be seen to emerge as the potential is swept to more positive potentials and can be attributed to the oxidation of other low weight species such as urate and ascorbate. The great advantage of the flavin group is that the peak is in a region where there are no competing processes.

The peak data from each of the urine samples were extracted, and the calibration data detailed in Fig. 7a used to determine the $\mathrm{pH}$. The results from the three samples are compared with the standard technique in Table 1.

It can be seen that the results are within $0.1 \mathrm{pH}$ unit of the standard system. Repetitive scanning within the urine did not degrade the response and indicates

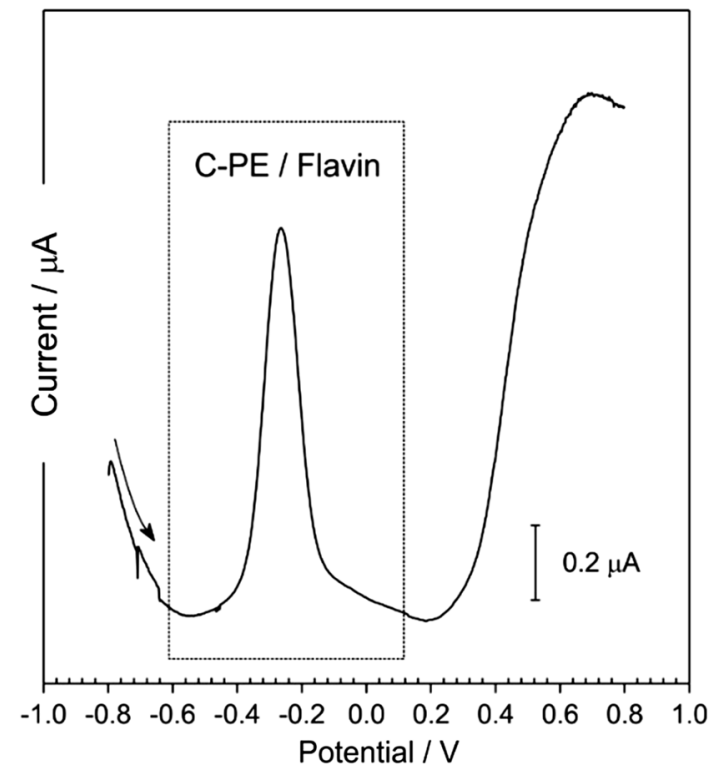

Figure 10 Square wave voltammogram detailing the response of a poly flavin modified carbon-polyethylene mesh electrode in human urine. that at least within urine, there is no cumulative fouling of the electrode. The fact that the flavin redox centre can be probed at very low potentials also avoids the possibility of oxidising matrix components such as tyrosine and tryptophan whose products are known to passivate electrode surfaces [30]. The structure of the underlying film is mechanically flexible, and it could be anticipated that it could serve as a versatile film for applications where there is a need to mould the electrode to contours of the prospective sample. While there are a multitude of approaches to measure the $\mathrm{pH}$ of urine, the spectroscopic methodologies in the present context are largely unsuitable for direct integration within ostomy appliance. The system exhibits a classical Nernst-like $(59 \mathrm{mV} / \mathrm{pH}$ ) response which is consistent with other solid-state devices employing both potentiometric [18-22] and voltammetric [25-27] sensing methodologies. It should be noted however that none of these system have been used in the measurement of $\mathrm{pH}$ within biological fluids. The system investigated here provides a number of key advantages over the existing approaches: the flavin redox centre lies in a region where there are few competing processes/interferences, the substrate is flexible and retains performance when bent or folded and the peak is robust. The latter is a critical advantage in that there is very little drift in the measurement of the peak potentialeither during normal scanning, after bending or after being fixed at perpendicular angle. The degree of error observed was less than $0.001 \mathrm{pH}$ units, and this was far superior to the potentiometric systems where drift is a common limitation.

\section{Conclusions}

The flavin redox film has been shown to be robust to repetitive scanning and can provide an accurate measure of $\mathrm{pH}$ in urine. The system is free from common interferences found in biofluids by virtue of the cathodic potentials necessary to interrogate the

Table 1 Determination of urine $\mathrm{pH}$ from healthy volunteers

\begin{tabular}{lll}
\hline Subject & Commercial probe $\mathrm{pH}$ & C-PE/flavin mesh $\mathrm{pH}$ \\
\hline F 1 & 5.98 & 5.89 \\
M 1 & 6.48 & 6.52 \\
M 2 & 6.35 & 6.45 \\
\hline
\end{tabular}


flavin redox centre, and, critically, the measurement of the oxidation potential can be achieved without the need for degassing. The latter should enable its adoption within a host of smart biomedical/textile applications where there is a need for mechanically flexible but electroanalytically sensitive sensors.

\section{Acknowledgements}

The authors are pleased to acknowledge financial support from the Department of Employment and Learning (DEL) Northern Ireland, EC-Lab Ltd, the British Council (DST-UKIERI: Ref 65/2017) and the University of Central Lancashire Innovation and Enterprise for supporting this work.

\section{Author Contributions}

AM was involved in ethics approval/human urine analysis and oversight of surface preparation and characterisation. CC contributed to electrochemical characterisation. AM was involved in physical characterisation/modification. CLL and CMT contributed to the synthesis of intermediates, RBS was involved in the supervision and characterisation of synthesis / products, JD contributed to the supervision of overall project and compilation of manuscript.

\section{Compliance with ethical standards}

Conflict of interest The authors confirm that there are no conflicts of interest arising in work presented here.

Open Access This article is distributed under the terms of the Creative Commons Attribution 4.0 International License (http://creativecommons.org/ licenses/by/4.0/), which permits unrestricted use, distribution, and reproduction in any medium, provided you give appropriate credit to the original author(s) and the source, provide a link to the Creative Commons license, and indicate if changes were made.

\section{References}

[1] Gray M, Colwell JC, Doughty D, Goldberg M, Hoeflok J, Manson A, McNichol L, Rao S (2013) Peristomal moisture- associated skin damage in adults with fecal ostomies. J Wound Ostomy Cont Nurs 40:389-399

[2] Gray M, Black JM, Baharestani MM, Bliss DZ, Colwell JC, Goldberg M, Kennedy-Evans KL, Logan S, Ratliff CR (2011) Moisture associated skin damage. J Wound Ostomy Cont Nurs 38:233-241

[3] Ratliff C (2010) Early peristomal skin complications reported by WOC nurses. J Wound Ostomy Cont Nurs 37:505-510

[4] Salvadalena GD (2013) The incidence of stoma and peristomal complications during the first 3 months after ostomy creation. J Wound Ostomy Cont Nurs 40:400-406

[5] Colwell JC, Ratliff CR, Goldberg M, Baharestani MM, Bliss DZ, Gray M, Kennedy-Evans KL, Logan S, Black JM (2011) MASD Part 3: peristomal moisture associated dermatitis and periwound moisture-associated dermatitis. J Wound Ostomy Cont Nurs 38:541-553

[6] Burch J (2008) Stoma care. Wiley, Hoboken

[7] Omura Y, Yamabe M, Anazawa S (2010) Peristomal skin disorder in patients with intestinal and urinary ostomies. J Wound Ostomy Cont Nurs 37:289-298

[8] Wells J, Doughty D (1994) Pouching principles and products. Ostomy Wound Manag 40:50-63

[9] Nybaek H, Lophagen S, Karlsmark T, Bang KD, Jemec G (2009) Stratum corneum integrity as a predictor for peristomal skin problems in ostomates. $\mathrm{Br} \mathrm{J}$ Dermatol 162:357-361

[10] Ali SM, Yosipovitch G (2013) Skin pH: from basic science to basic skin care, acta derm. Venerol 93:261-267

[11] Leyden JJ, Stewart R, Kligman AM (1979) Updated in vivo methods for evaluating topical antimicrobial agents on human skin. J Invest Dermatol 72:165-170

[12] Korting HC, Hubner K, Greiner K, Hamm G, Braun-Falco O (1990) Differences in the skin surface ph and bacterial microflora due to the long term application of synthetic detergent preparation of $\mathrm{pH} 5.5$ and $\mathrm{pH}$ 7.0. results of a crossover trial in healthy volunteers. Acta Dermatol Venereol $70: 429-431$

[13] Rippke F, Schreiner V, Schwanitz HJ (2002) The acidic milieu of the horny layer: new findings on the physiology and pathophysiology of skin $\mathrm{pH}$. Am J Clin Dermatol Res 3:261-272

[14] Patel ND, Ward RD, Calle J, Remer EM, Monga M (2017) Vascular disease and kidney stones: abdominal aortic calcifications are associated with low urine ph and hypocitraturia. J Endourol 31:956-961

[15] Stickler DJ (2014) Clinical complications of urinary catheters caused by crystalline biofilms: something needs to be done. J Intern Med 276:120-129 
[16] Manissorn J, Fong-ngern K, Peerapen P, Thongboonkerd V (2017) Systematic evaluation for effects of urine $\mathrm{pH}$ on calcium oxalate crystallization, crystal-cell adhesion and internalization into renal tubular cells. Scientific Reports, Article Number: 1798

[17] Parmenter BH, Slater GJ, Frassetto LA (2017) Spot-testing urine $\mathrm{pH}$, a novel dietary biomarker? A randomised crossover trial. Nutr Dietics 74:313-319

[18] Santos L, Neto JP, Crespo A, Nunes D, Costa N, Fonseca IM, Barquinha P, Silva J, Fortunato Martins RE, Pereira L (2014) $\mathrm{WO}_{3}$ nanoparticle-based conformable $\mathrm{pH}$ sensor. ACS Appl Mater Interfaces 6:12226-12234

[19] Qingwen L, Yiming W, Guoan L (1999) pH-response of nanosized $\mathrm{MnO}_{2}$ prepared with solid state reaction route at room temperature. Sens Actuators B 59:42-47

[20] Sardarinejad A, Maurya D, Alameh K (2015) The pH sensing properties of $\mathrm{RF}$ sputtered $\mathrm{RuO}_{2}$ thin-film prepared using different $\mathrm{Ar} / \mathrm{O}_{2}$ flow ratio. Materials 8:3352-3363

[21] Huang WD, Cao H, Deb S, Chiao M, Chiao JCA (2011) Flexible $\mathrm{pH}$ sensor based on the iridium oxide sensing film. Sens Actuators A 169:1-11

[22] Maiolo L, Mirabella S, Maita F, Alberti A, Minotti A, Strano V, Pecora A, Shacham-Diamand Y, Fortunato G (2014) Flexible $\mathrm{pH}$ sensors based on polysilicon thin film transistors and $\mathrm{ZnO}$ nanowalls. Appl Phys Lett 105:093501

[23] Salvo P, Calisi N, Melai B, Cortigiani B, Mannini M, Caneschi A, Lorenzetti G, Paoletti C, Lomonaco T, Paolicchi A, Scataglini I, Dini V, Romanelli M, Fuoco R, Di Francesco F (2017) Temperature and $\mathrm{pH}$ sensors based on graphenic materials. Biosens Bioelectron 91:870-877

[24] Yang J, Kwak TJ, Zhang X, McClain R, Chang W-J, Gunasekaran S (2016) Digital $\mathrm{pH}$ test strips for in-field $\mathrm{pH}$ monitoring using iridium oxide-reduced graphene oxide hybrid thin films. ACS Sens 1:1235-1243

[25] Lafitte VGH, Wang WX, Yashina AS, Lawrence NS (2008) Anthraquinone-ferrocene film electrodes: utility in $\mathrm{pH}$ and oxygen sensing. Electrochem Commun 12:1831-1834

[26] Streeter I, Leventis HC, Wildgoose GG, Pandurangappa M, Lawrence NS, Jiang L, Jones TG, Compton RG (2004) A sensitive reagentless $\mathrm{pH}$ probe with a ca. $120 \mathrm{mV} / \mathrm{pH}$ unit response. J Solid State Electrochem 8:718-721

[27] Xiong L, Batchelor-McAuley C, Compton RG (2011) Calibrationless $\mathrm{pH}$ sensors based on nitrosophenyl and ferrocenyl co-modified screen printed electrodes. Sens Actuators B 159:251-255

[28] Anderson A, Phair J, Benson J, Meenan BJ, Davis J (2014) Investigating the use of endogenous quinoid moieties on carbon fibre as means of developing micro $\mathrm{pH}$ sensors. Mater Sci Eng C 43:533-537
[29] Phair J, Davis J, Leach C, Cardosi MF (2013) Atmospheric pressure plasma treated carbon fibre weave: a flexible approach to wound monitoring. Electrochem Commun 33:99-101

[30] Phair J, Benson J, McCormac C, Cundell J, Gracheva S, Wilkinson D, Forsythe S, Davis J (2014) Butyl grafted polyethylene films doped with carbon black: a foundation for the development of smart bandages. Sens Actuators B 193:764-769

[31] Nyein HYY, Gao W, Shahpar Z, Emaminejad S, Challa S, Chen K, Fahad HM, Tai L-C, Ota H, Davis RW, Javey A (2016) A wearable electrochemical platform for noninvasive simultaneous monitoring of $\mathrm{Ca}_{2+}$ and $\mathrm{pH}$. ACS Nano 10:7216-7224

[32] Nakata S, Arie T, Akita S, Takei K (2017) Wearable, Flexible, and multifunctional healthcare device with an ISFET chemical sensor for simultaneous sweat $\mathrm{pH}$ and skin temperature monitoring. ACS Sens 2:443-448

[33] Jin J, Lin Y, Song M, Gui C, Leesirisan S (2013) Enhancing the electrical conductivity of polymer composites. Eur Polym J 49:1066-1072

[34] Valipour A, Roushani M (2017) Using silver nanoparticle and thiol graphene quantum dots nanocomposite as a substratum to load antibody for detection of hepatitis $\mathrm{C}$ virus core antigen: electrochemical oxidation of riboflavin was used as redox probe. Biosens Bioelectron 89:946-951

[35] Casimero C, McConville A, Fearon JJ, Lawrence CL, Taylor CM, Smith RB, Davis J (2018) Sensor systems for bacterial reactors: a new flavin-phenol composite film for the in situ voltammetric measurement of $\mathrm{pH}$. Anal Chim Acta 1027:1-8

[36] Hegarty C, Kirkwood S, Cardosi MF, Lawrence CL, Taylor CM, Smith RB, Davis J (2018) Disposable solid state $\mathrm{pH}$ sensor based on flavin polymer-ferrocyanide redox couples. Microchem J 139:210-215

[37] Roushani M, Abdi Z (2014) Novel electrochemical sensor based on graphene quantum dots/riboflavin nanocomposite for the detection of persulfate. Sens Actuators B 201:503-510

[38] Bejugam M, Sewitz S, Shirude PS, Rodriguez R, Shahid R, Balasubramanian S (2007) Trisubstituted isoalloxazines as a new class of g-quadruplex binding ligands: small molecule regulation of c-kit oncogene expression. J Am Chem Soc 129:12926-12927

Publisher's Note Springer Nature remains neutral with regard to jurisdictional claims in published maps and institutional affiliations. 